\title{
Multiobjective Traffic Signal Control Model for Intersection Based on Dynamic Turning Movements Estimation
}

\author{
Pengpeng Jiao and Tuo Sun \\ Beijing Urban Transportation Infrastructure Engineering Technology Research Center, Beijing University of Civil Engineering and \\ Architecture, Beijing 100044, China
}

Correspondence should be addressed to Pengpeng Jiao; jiaopengpeng@bucea.edu.cn

Received 22 July 2014; Accepted 15 September 2014; Published 29 September 2014

Academic Editor: Valentina Emilia Balas

Copyright (C) 2014 P. Jiao and T. Sun. This is an open access article distributed under the Creative Commons Attribution License, which permits unrestricted use, distribution, and reproduction in any medium, provided the original work is properly cited.

The real-time traffic signal control for intersection requires dynamic turning movements as the basic input data. It is impossible to detect dynamic turning movements directly through current traffic surveillance systems, but dynamic origin-destination (OD) estimation can obtain it. However, the combined models of dynamic O-D estimation and real-time traffic signal control are rare in the literature. A framework for the multiobjective traffic signal control model for intersection based on dynamic O-D estimation (MSC-DODE) is presented. A state-space model using Kalman filtering is first formulated to estimate the dynamic turning movements; then a revised sequential Kalman filtering algorithm is designed to solve the model, and the root mean square error and mean percentage error are used to evaluate the accuracy of estimated dynamic turning proportions. Furthermore, a multiobjective traffic signal control model is put forward to achieve real-time signal control parameters and evaluation indices. Finally, based on practical survey data, the evaluation indices from MSC-DODE are compared with those from Webster method. The actual and estimated turning movements are further input into MSC-DODE, respectively, and results are also compared. Case studies show that results of MSC-DODE are better than those of Webster method and are very close to unavailable actual values.

\section{Introduction}

Real-time traffic signal control system is most important for alleviating urban traffic congestion problems. As the basic input data of traffic signal control models, traffic counts at intersections are time-variant, nonlinear, and random. Therefore, to achieve the real-time link counts of entrance and exiting legs, as well as the turning movements at the intersection, is the key for decision of the traffic signal control scheme. Under the existing traffic surveillance systems, it is impossible to obtain the turning movements of intersections directly. However, based on the detected time-series of link counts of entrance and exiting legs, the real-time turning movements can be obtained using dynamic origin-destination (O-D) flows estimation (DODE) technique. Therefore, the traffic signal control model based on DODE is most important for the optimization of intersection signal control schemes.

Existing researches about dynamic O-D estimation can be generally classified into three categories according to the target network: for intersection, for freeway segment, and for general road network.
For intersection, Cremer and Keller [1] first used the timeseries of link counts at entering and exiting legs as input data and formulated a least-square model to estimate the turning movement flows at intersection. Nihan and Davis [2] further put forward a recursive least-square formulation using the measurements of all past intervals and considered the reduction of early intervals. Nihan and Davis [3] presented a maximum likelihood estimation model, as well as an expectancy maximization algorithm. Bell [4] proposed a revised optimization model considering the platoon dispersion between entering and exiting flows. Li and De Moor [5] further introduced the equality constraint and formulated a new least-square model. Jiao et al. [6] noticed that the leastsquare formulation will enlarge the occasional outliers of input flows, presented an optimization model to minimize the sum of absolute deviations between observed and estimated traffic counts, and designed a genetic algorithm for solution. Based on the above work, Jiao et al. [7] further proposed a real-time arterial coordination control method. Moreover, Jiao et al. $[8,9]$ put forward a Bayesian combined model to 
estimate time-varying turning movement flows at intersections, as well as a bi-Bayesian combined framework for twostep prediction of such flows. Without considering dynamic travel time and route choice of vehicles, the above models are only applicable for intersections.

Introduction of dynamic travel time extended intersection models to freeway segment. Chang and $\mathrm{Wu}$ [10] first formulated a state-space model using Kalman filtering method. Sherali et al. [11] introduced the travel time to conventional least-square models and designed a projected conjugate gradient method for solving. Lin and Chang [12] further employed the dynamic travel time in the state-space model and put forward an efficient and robust on-line estimation method. Li et al. [13] formulated a nonlinear statespace model using unscented Kalman filtering. Considering dynamic travel time but without route choice, the above models are applicable for freeway segment.

Further introduction of dynamic route choice extended freeway segment models to general road network. Okutani [14] employed a strengthened state variable including all past intervals and put forward a network estimation model based on freeway segment models. Ashok and Ben-Akiva [15] used deviations of O-D flows from prior estimates based on corresponding historical data as state variables, improved the system observability, and revised Okutani's model [14]. Ashok and Ben-Akiva [16] further put forward an estimation model integrating random dynamic traffic assignment, solved the problem that assignment matrix was unavailable in conventional models, and considered the random travel demand. Zhou and Mahmassani [17] also employed O-D deviations as state variables and formulated an estimation method based on self-learning mechanism. To avoid the errors inherent in the dynamic route choice, Sherali and Park [18] used real-time path flows as state variables and presented an optimization model. Along another line, Nie and Zhang [19] introduced the variational inequality method to the DODE problem and formulated a series of new models. More recently, Cheng et al. [20] proposed a Bayesian network model for O-D matrices estimation using prior and some observed link flows, but it was for static condition. Considering both dynamic travel time and route choice, most of the above models are applicable for general road network.

With respect to the researches on traffic signal control for intersections, genetic reinforcement learning [21], fuzzy computing [22], artificial neural network (ANN) [23], and genetic algorithm [24] methods have been extensively studied over the past two decades, and many signal control models based on historical information have been formulated, as well as the revised Webster method [25] and many multiobjective optimization methods [26]. Under the signal control, the delay, queue length, and capacity have been improved greatly. However, the real-time turning movements, which are most important but cannot be detected directly, are still left unused.

To the best of our knowledge, there is no existing research which takes the real-time turning movements estimation into account in the traffic signal control model. Therefore, the key feature of this paper is to employ the estimated turning movements in the signal control at intersections.

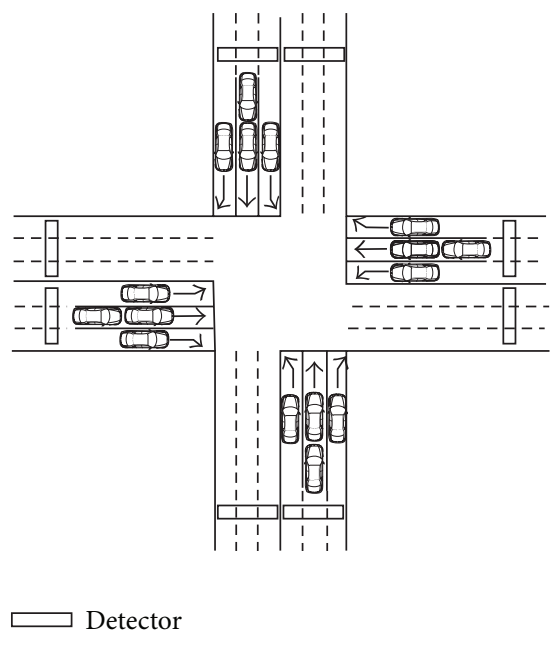

FIGURE 1: Topology of a typical intersection.

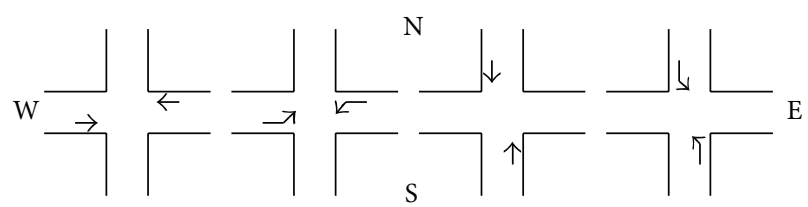

FIGURE 2: Signal phase of the intersection.

The rest of this paper is organized as follows. The basic problem statement, as well as the interrelation between dynamic O-D estimation and signal control, is presented in Section 2. A Kalman filtering based state-space model is formulated to estimate real-time turning movements in Section 3. A multiobjective traffic signal control model using estimated turning movements as input data is put forward in Section 4. Two algorithms are designed to solve the above two models, respectively, in Section 5. The evaluation results based on practical traffic data are reported in Section 6. Conclusions and further researches are summarized in the last section.

\section{Problem Statement}

For a typical intersection with $r$ entrance legs and $s$ exiting legs, detectors are placed at all legs to get the entering and exiting link flows, as shown in Figure 1.

This paper uses a 4-phase signal control for the intersection, as shown in Figure 2. There is no right-turn restriction in the timing plan.

Combining the DODE model based on Kalman filtering and the signal control model based on multiobjective optimization together, this paper puts forward a multiobjective signal control model based on DODE (MSC-DODE). The interrelation between dynamic O-D flows and signal control is shown in Figure 3.

This model takes the estimated turning movement proportions $B_{i j}(k)$ as input data for the signal control and aims to realize the real-time signal control and to achieve the comprehensive optimization of delay time, queue length, and effective capacity. 


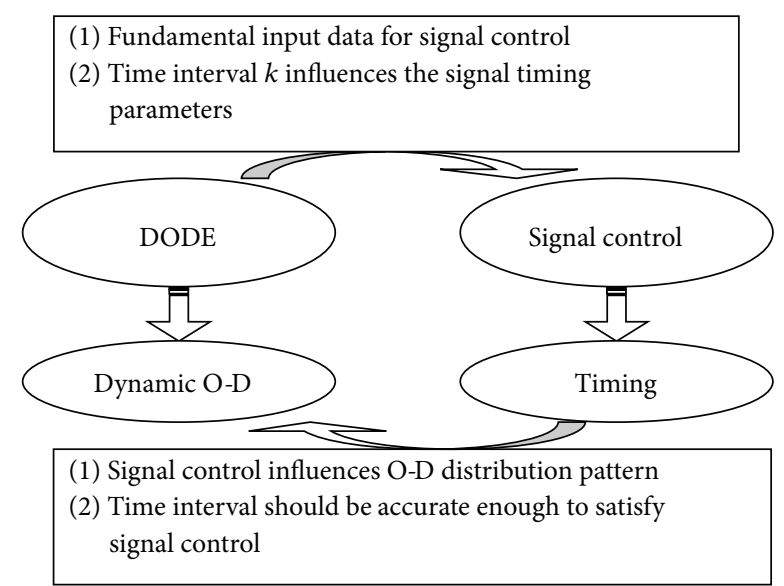

FIGURE 3: Interrelation between dynamic O-D and signal control.

\section{Dynamic Turning Movements Estimation for Intersection}

To meet the accuracy and efficiency needs for on-line application, a Kalman filtering based state-space model is used to estimate the dynamic turning movement proportions.

We define variables in this model as follows:

$Q_{i}(k)$ : entering link flows from leg $i$ during interval $k$, $i=1,2, \ldots, r$;

$Y_{j}(k)$ : exiting link flows from leg $j$ during interval $k$, $j=1,2, \ldots, s$;

$T_{i j}(k)$ : turning flows from leg $i$ during interval $k$ to leg $j$, that is, the time-dependent turning movements or dynamic O-D flows;

$B_{i j}(k)$ : turning movement fraction, that is, split parameter: it is equal to the ratio of $Q_{i}(k)$ turning from leg $i$ to leg $j$; obviously, $B_{i j}(k)=T_{i j}(k) / Q_{i}(k)$, $0 \leq B_{i j}(k) \leq 1, \sum_{j} B_{i j}(k)=1$.

Since we can obtain $Q_{i}(k)$ and $Y_{j}(k)$ in real-time from traffic detectors placed at entrance and exiting legs, the key point of this section can be represented as how to formulate a model to estimate the time-dependent turning movement fractions $B_{i j}(k)$ from the detected time-series of link counts $Q_{i}(k)$ and $Y_{j}(k)$.

The split parameter $B_{i j}(k)$ is used as the state variable to reflect the interrelation between the link counts of entrance and exiting legs; then the state transition equation is represented as

$$
\mathbf{B}(k)=\mathbf{B}(k-1)+\mathbf{W}(k),
$$

where $\mathbf{B}(k)$ is the vector form of $B_{i j}(k)$ and $\mathbf{W}(k)$ is a vector of random errors. It is a column Gaussian white noise vector with zero mean and covariance $\mathbf{D} \delta_{k l}$, where $\mathbf{D}$ is a constant semipositive matrix and $\delta_{k l}$ is the Kronecker delta; that is, $\delta_{k l}=1$ when $k=l$; otherwise $\delta_{k l}=0$.
Assuming there is no U-turn at the intersection, that is, $B_{i i}(k)=0$, then the measurement equation can be represented as

$$
\mathbf{Y}(k)=\mathbf{Q}(k) * \mathbf{B}(k)+\mathbf{e}(k),
$$

where $\mathbf{Y}(k)$ is a column vector of link counts at exiting legs, $\mathbf{Q}(k)$ is the measurement matrix, $\mathbf{e}(k)$ is the column vector of link volume measurement errors with zero mean and covariance $\mathbf{R} \delta_{k l}$, and $\mathbf{R}$ is also a constant semipositive matrix.

\section{Traffic Signal Control Model Based on Dynamic O-D Estimation}

The notations used in this model description are defined in the Notation section.

The displayed green time is defined as

$$
g_{x}=G_{x}-A_{x}+l_{x}
$$

In order to optimize the signal timing plan, a multiobjective optimization model is formulated, and three indices are integrated to evaluate the model, including delay time, queue length, and effective capacity. Based on the estimated dynamic turning movements in Section 3 and the detected saturation headways, $s_{x}, q_{x}, V, p_{x}, P$, and $\alpha$ can be determined. These parameters are the basis to solve the optimization model.

The abovementioned three evaluation indices are defined as follows.

(1) Average delay time of arrival vehicles during phase $x$ : $d_{x}$.

It is equal to the difference between the travel time with and without control devices:

$$
d_{x}=\frac{T\left(1-G_{x} / T\right)^{2}}{2\left(1-p_{x}\right)}+\frac{\left(1-\left(\sum_{x=1}^{n} l_{x}\right) / T\right)^{2}}{2\left(\left(\sum_{x=1}^{n} l_{x}\right) / T\right)},
$$

where $n$ is the number of phases during a cycle (4 in this paper).

(2) Average queue length during phase $x: L_{x}$.

It is equal to the average value of maximum queue length of each lane from the start of green phase:

$$
L_{x}= \begin{cases}\frac{2 q_{x} R+0.25 V(\alpha-1)}{\alpha} & \text { saturation } \\ 2 q_{x} R & \text { unsaturation. }\end{cases}
$$

(3) Effective capacity during phase $x: Q_{x}$.

It is a most important index for intersection and denotes the total saturation flow during all effective green times during a certain period:

$$
Q_{x}=\lambda_{x} s_{x}
$$


To integrate the above three indices together, three weighting coefficients, $K_{x}^{1}, K_{x}^{2}$, and $K_{x}^{3}$, are defined as

$$
\begin{gathered}
K_{x}^{1}=2 s_{x} p_{x}(1-P), \\
K_{x}^{2}=s_{x} p_{x}(1-P) T, \\
K_{x}^{3}=2(3600 / T) P .
\end{gathered}
$$

Equation (7) reflects the following influences.

(1) $K_{x}^{1}$ and $K_{x}^{2}$ decrease with the increase of $P$, while $K_{x}^{3}$ increases with the increase of $P$. The objective is to place the optimization emphasis on delay time and queue length during nonpeak hours, while it is placed on effective capacity during peak hours.

(2) Introduction of $T$ into $K_{x}^{2}$ means that the weight of queue length increases with the extension of cycle length.

Based on the above three weighting coefficients, the nonlinear optimization model is formulated as follows, with cycle and effective green time as the independent variables and with minimum delay, minimum queue length, and maximum capacity as the objectives:

$$
\begin{array}{ll}
\min & f\left(G_{x}, T\right)=\sum_{x=1}^{n}\left[K_{x}^{1} d_{x}+K_{x}^{2} L_{x}-K_{x}^{3} Q_{x}\right] \\
\text { s.t. } & \sum_{x=1}^{n}\left(G_{x}+A_{x}+R_{x}\right)=T \\
& 0.9 p_{x} \leq \frac{G_{x}}{T} \leq 1.1 p_{x}, \quad 1 \leq x \leq n \\
& \text { green }_{x, \min } \leq G_{x} \leq \text { green }_{x, \max }, \quad 1 \leq x \leq n \\
& \sum_{x=1}^{n} G_{x}+L \leq J, \quad 1 \leq x \leq n \\
& G_{x} \geq 0, \quad 1 \leq x \leq n,
\end{array}
$$

where green $_{x, \text { min }}$ is the minimum green time during phase $x$ (15 seconds in this paper) and green $x_{x, \max }$ is the maximum green time during phase $x$ (60 seconds in this paper).

In this model, $p_{x}$ and $P$ are obtained based on the estimated dynamic turning movements proportions $B_{i j}(k)$, which reflect the relation between DODE and traffic signal control.

\section{Algorithm Design}

5.1. Estimation Algorithm for Intersection Turning Movement Proportions. An efficient sequential Kalman filtering algorithm is designed to estimate the dynamic turning movement proportions, and the truncation and normalization process [2] are used to modify the results to satisfy the inherent constraints of $B_{i j}(k)$ in its definition. A step-by-step description of the estimation algorithm for the proposed model is presented below.
Step 1. Initialization:

(1) generate the initial values $B_{i j}(0)$ of $B_{i j}(k)$;

(2) $\mathbf{P}(0)=\operatorname{var}[\mathbf{B}(0)]$;

(3) $\operatorname{var}[\mathbf{e}(k)]=\operatorname{diag}\left[R_{1}, R_{2}, \ldots, R_{r}\right]$;

(4) $\operatorname{var}[\mathbf{W}(k)]=\operatorname{diag}\left[D_{1}, D_{2}, \ldots, D_{r s}\right]$.

Step 2. Assume $k=1$, and compute the measurement matrix $\mathbf{Q}(k)$; then define $\mathbf{f}_{i}$ as a row vector of $\mathbf{Q}(k)$ in row $i$.

Step 3. Initialize the Kalman filtering:

(1) $\mathbf{B}^{0}=\mathbf{B}(k-1)$;

(2) $\mathbf{P}^{0}=\mathbf{P}(k-1)+\mathbf{D}$.

Step 4. When $i=1, \ldots, r$, perform the Kalman filtering recursion, truncation, and normalization:

(1) $\mathbf{g}^{i}=\mathbf{P}^{i-1} \mathbf{f}_{i}^{T}\left[\mathbf{f}_{i} \mathbf{P}^{i-1} \mathbf{f}_{i}^{T}+R_{i}\right]^{-1}$;

(2) $\mathbf{P}^{i}=\mathbf{P}^{i-1}-\mathbf{g}^{i} \mathbf{f}_{i} \mathbf{P}^{i-1}$;

(3) $\delta^{i}=Y_{i}(k)-\mathbf{f}_{i} \mathbf{B}^{i-1}$;

(4) truncation: compute $\alpha^{\prime}$, where $\alpha^{\prime}=\max _{0 \leq \alpha \leq 1}[\alpha \mid 0 \leq$ $\left.\mathbf{B}^{i-1}+\alpha \delta^{i} \mathbf{g}^{i} \leq 1\right]$; then assume $\mathbf{B}^{i}=\mathbf{B}^{i-1}+\alpha^{\prime} \delta^{i} \mathbf{g}^{i} ;$

(5) normalization: when $m=1,2, \ldots, r$, assume $\beta_{m}=$ $\sum_{j=1}^{s} B_{m j}^{i}$; then $B_{m j}^{i}=B_{m j}^{i} / \beta_{m}, j=1,2, \ldots, s$.

Step 5. Assume $\mathbf{P}(k)=\mathbf{P}^{r}$ and $\mathbf{B}(k)=\mathbf{B}^{r}$; then return to Step 2 for the next round of iteration.

In the above algorithm, we improved the calculation efficiency by using the sequential Kalman filtering technique to avoid inverse matrix computation. Additionally, $B_{i i}(k)$ is assumed to be 0 to neglect the U-turn phenomena at the intersection, and we incorporated it in the algorithm programing using $\mathrm{M}$ language of Matlab software.

5.2. Optimization for MSC-DODE Model. A normal nonlinear optimization algorithm is developed to solve the multiobjective signal control model to achieve the timing plan parameters and evaluation indices, which is implemented using Lingo software.

\section{Case Study}

To testify the accuracy and efficiency of the MSC-DODE model, we collected the field data of 3 hours through a survey during morning peak hour at Zhaodengyu-Pinganli west intersection in Beijing city and obtained enough data for the case study, including time-dependent link counts on all entrance and exiting legs, delay time, queue length, and saturation headway. Furthermore, we implemented the algorithm in Section 5 and achieved all estimated turning movement proportions and signal timing parameters. For the purpose of accuracy, a time interval of 2 minutes is used in the DODE model; therefore there are totally 90 intervals. 


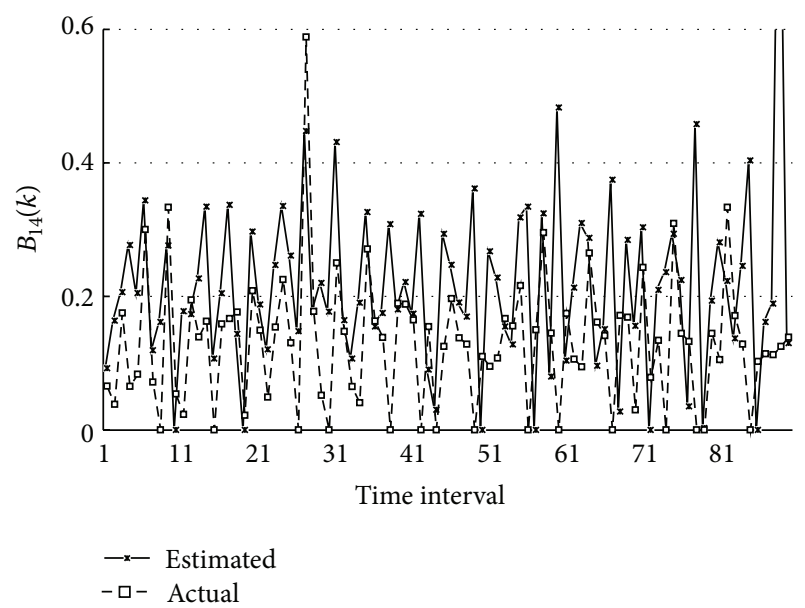

Figure 4: Comparison between estimated and actual $B_{14}(k)$.

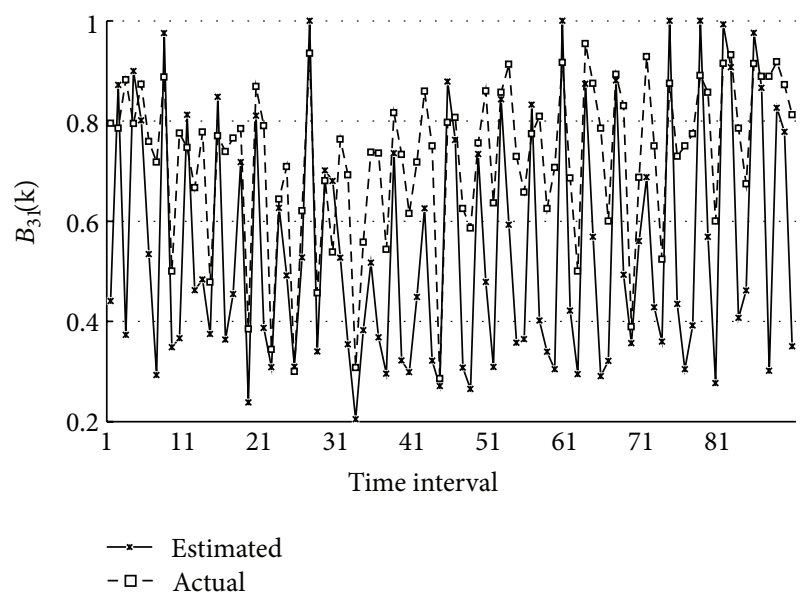

Figure 5: Comparison between estimated and actual $B_{31}(k)$.

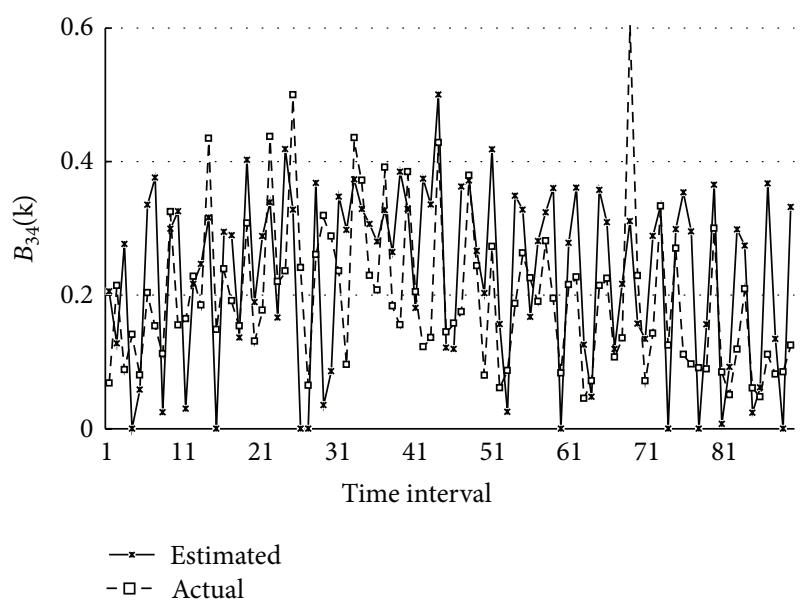

FIGURE 6: Comparison between estimated and actual $B_{34}(k)$.

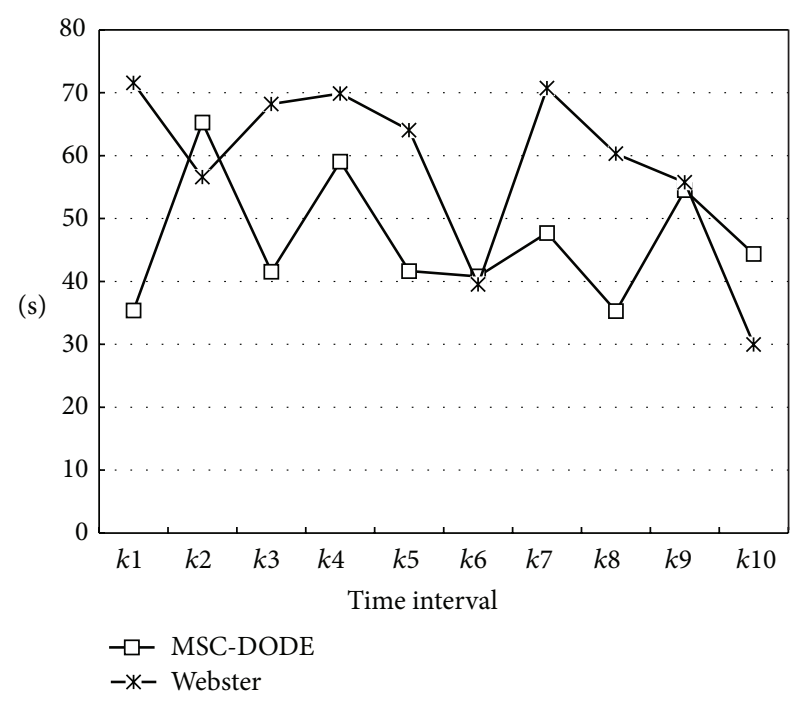

Figure 7: Delay time comparison between MSC-DODE and Webster method.

6.1. Comparison between Estimated and Actual Turning Movement Proportions. Taking the right-turn at east entrance, go straight at west entrance and left-turn at west entrance, for instance, that is, $B_{14}(k), B_{31}(k)$, and $B_{34}(k)$; the comparison results are illustrated in Figures 4, 5, and 6. We can further find out that the root mean square error (RMSE) range is below 0.1 and the mean percentage error is no more than about 0.01 . Here results of the first five intervals are excluded from the RMSE and mean percentage error statistics because the estimation results of Kalman filtering during its initialization period are usually unreliable. Obviously the estimation results are accurate enough to support traffic signal control systems.

6.2. Comparison between MSC-DODE and Webster Method. To reflect the effectiveness of MSC-DODE model, 10 sets of data were used to achieve the signal parameters using MSCDODE model and classical Webster method, respectively. The results of three evaluation indices are reported in Figures 7-9, including delay time, queue length, and effective capacity.

From Figures 7, 8, and 9 we can find out the following results:

(1) total sum and average delay time of MSC-DODE model are both much less than those of Webster model;

(2) total sum and average queue length of MSC-DODE model are both much less than those of Webster model;

(3) total sum and average effective capacity of MSCDODE model are both greater than those of Webster model.

In a word, the signal timing plan from MSC-DODE model is much more optimized than that from Webster method. 


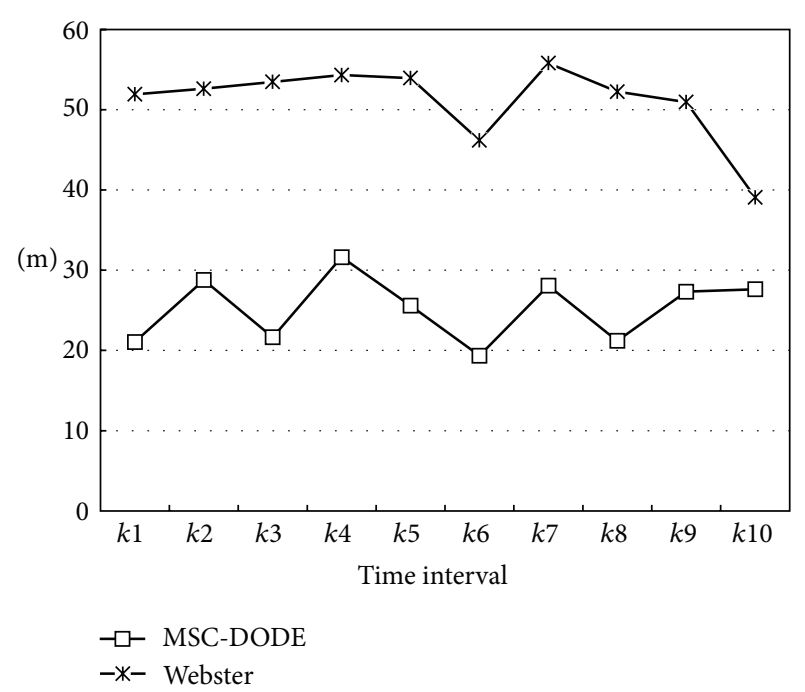

FIGURE 8: Queue length comparison between MSC-DODE and Webster method.

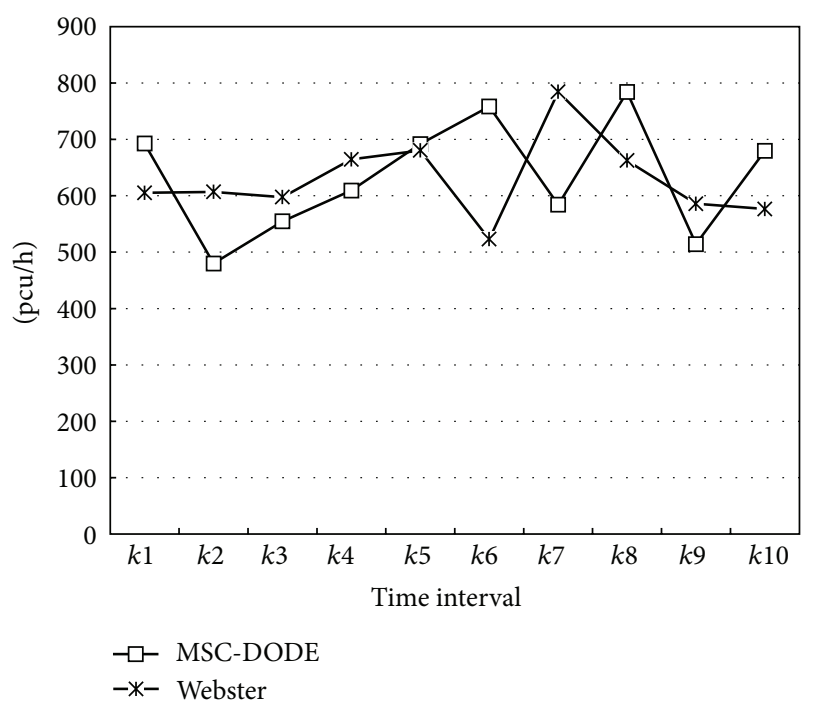

FIGURE 9: Effective capacity comparison between MSC-DODE and Webster method.

6.3. Comparison between Inputting Actual and Estimated Turning Movements. To further testify the influences of DODE results on signal control, we input the estimated and actually collected turning movements (unavailable in realtime systems) into the MSC-DODE model, respectively, and the comparison results are shown in Figures 10, 11, and 12.

From the above figures we can find out that the results of inputting estimated values are much close to and sometimes even better than those of inputting actual values. Meanwhile the changing trends are much similar.

Finally, the above case studies show that MSC-DODE model is much accurate and efficient for intersection signal control, especially under the current condition that it impossible to detect dynamic turning movements directly through traffic surveillance systems.

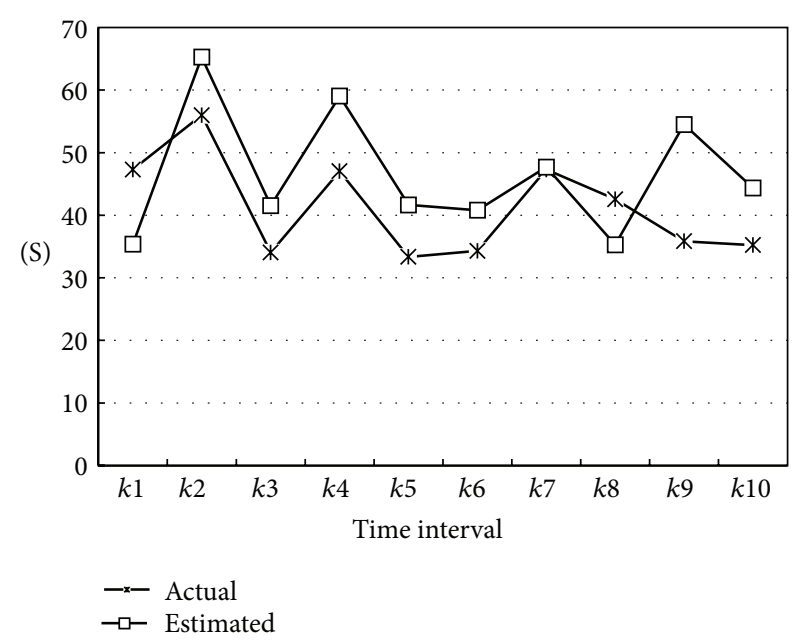

FIGURE 10: Delay time comparison between inputting estimated and actual turning movements.

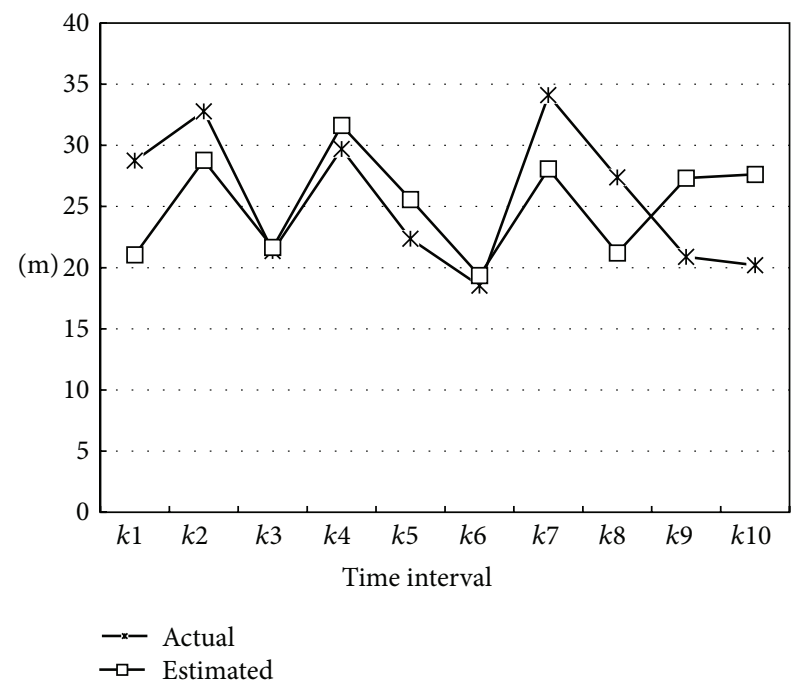

FIGURE 11: Queue length comparison between inputting estimated and actual turning movements.

\section{Conclusions}

In this paper, we integrate dynamic turning movements estimation and signal control together and put forward a multiobjective traffic signal control model based on dynamic O-D flows estimation (MSC-DODE). To optimize the delay time, queue length, and effective capacity together, this model is not only capable of taking advantage of intersection turning movements to further improve the effects of signal control, but also able to capture the temporal interdependency among turning movements and link counts. The reported examples have demonstrated that the proposed model is much more outstanding than classical Webster method and is accurate and efficient enough for on-line traffic signal control application. Furthermore, the signal timing plans from MSC-DODE model remain time-varying; that is, this paper presents a realtime signal control framework. 


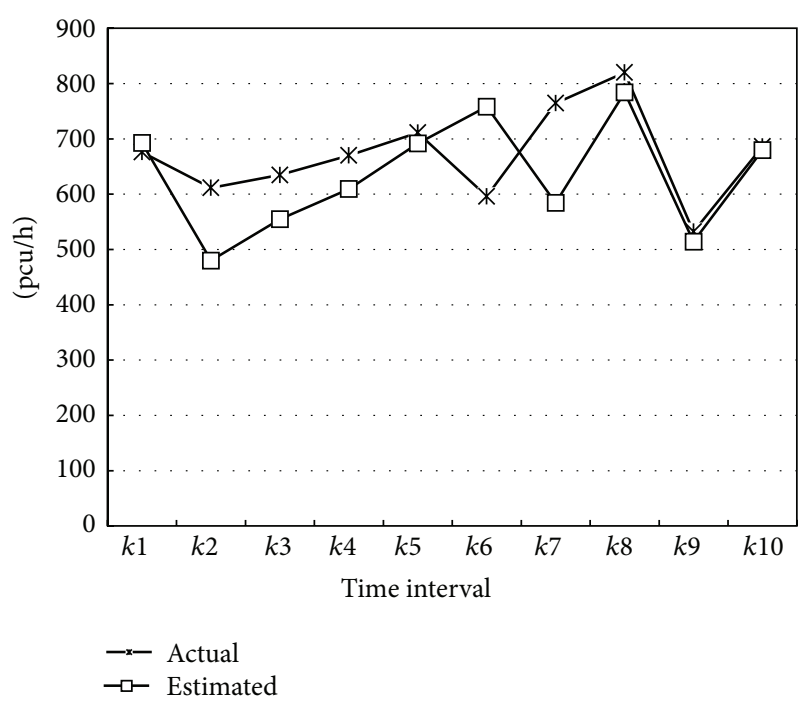

FIGURE 12: Effective capacity comparison between inputting estimated and actual turning movements.

Further researches are directed towards two aspects. The first is to take into account the travel time of intersection turning vehicles under traffic congestion. The second is to consider the influences of upstream and downstream intersections and formulate a model of urban arterial coordination control.

\section{Notations}

$T$ : Signal cycle length of the intersection

$s_{x}$ : Saturation flow during phase $x$

$q_{x}$ : Arrival flow rate during phase $x$

$V$ : Traffic volume

$L$ : Total lost time during a signal cycle (16 seconds in this paper)

$\lambda_{x}$ : Split of phase $x$

$l_{x}$ : Start-up lost time of phase $x$ ( 3 seconds in this paper)

$g_{x}$ : Displayed green time of phase $x$

$G_{x}$ : Effective green time of phase $x$

$A_{x}$ : Yellow change interval of phase $x(3$ seconds in this paper)

$R_{x}$ : Red clearance interval of phase $x$ ( 3 seconds in this paper)

$J$ : Maximum cycle length (180 seconds in this paper)

$d_{x}$ : Average delay time of arrival vehicles during phase $x$

$L_{x}$ : Average queue length during phase $x$

$Q_{x}$ : Effective capacity during phase $x$

$p_{x}$ : Volume-to-saturation flow ratio during phase $x$

$P:$ Sum of $p_{x}$ during a signal cycle

$R$ : Red time

$\alpha$ : Volume-to-capacity ratio.

\section{Conflict of Interests}

The authors declare that there is no conflict of interests regarding the publication of this paper.

\section{Acknowledgments}

The research herein reported has been supported by the National Natural Science Foundation of China Project (51208024), Science and Technology Project of Ministry of Housing and Urban-Rural Development of China (2013-K56), Beijing Philosophy and Social Science Project (14CSC014), Excellent Talents Project of Beijing Municipal Committee Department of Organization (2013D005017000001), and the Importation and Development of High-Caliber Talents Project of Beijing Municipal Institutions (CIT\&TCD201404071).

\section{References}

[1] M. Cremer and H. Keller, "A systems dynamics approach to the estimation of entry and exit O-D flows," in Proceedings of the 9th International Symposium on Transportation and Traffic Theory, pp. 431-450, VUN Science Press, Utrecht, The Netherlands, 1984.

[2] N. L. Nihan and G. A. Davis, "Recursive estimation of origindestination matrices from input/output counts," Transportation Research Part B, vol. 21, no. 2, pp. 149-163, 1987.

[3] N. L. Nihan and G. A. Davis, "Application of prediction-error minimization and maximum likelihood to estimate intersection O-D matrices from traffic counts," Transportation Science, vol. 23, no. 2, pp. 77-90, 1989.

[4] M. G. H. Bell, "The real time estimation of origin-destination flows in the presence of platoon dispersion," Transportation Research B, vol. 25, no. 2-3, pp. 115-125, 1991.

[5] B. Li and B. De Moor, "Recursive estimation based on the equality-constrained optimization for intersection origindestination matrices," Transportation Research Part B: Methodological, vol. 33, no. 3, pp. 203-214, 1999.

[6] P. Jiao, H. Lu, and L. Yang, "Real-time estimation of turning movement proportions based on genetic algorithm," in Proceedings of the 8th International IEEE Conference on Intelligent Transportation Systems, pp. 484-489, Vienna, Austria, September 2005 .

[7] P. Jiao, H. Wang, and T. Sun, "Real-time arterial coordination control based on dynamic intersection turning fractions estimation using genetic algorithm," Mathematical Problems in Engineering, vol. 2014, Article ID 430497, 10 pages, 2014.

[8] P. Jiao, T. Sun, and L. Du, "A Bayesian combined model for time-dependent turning movement proportions estimation at intersections," Mathematical Problems in Engineering, vol. 2014, Article ID 607195, 8 pages, 2014.

[9] P. Jiao, M. Liu, J. Guo, and T. Sun, "Bi-Bayesian combined model for two-step prediction of dynamic turning movement proportions at intersections," Advances in Mechanical Engineering, vol. 2014, Article ID 439031, 9 pages, 2014.

[10] G.-L. Chang and J. Wu, "Recursive estimation of time-varying origin-destination flows from traffic counts in freeway corridors," Transportation Research Part B, vol. 28, no. 2, pp. 141-160, 1994. 
[11] H. D. Sherali, N. Arora, and A. G. Hobeika, "Parameter optimization methods for estimating dynamic origin-destination trip-tables," Transportation Research Part B: Methodological, vol. 31, no. 2, pp. 141-157, 1997.

[12] P.-W. Lin and G.-L. Chang, "A generalized model and solution algorithm for estimation of the dynamic freeway origindestination matrix," Transportation Research Part B: Methodological, vol. 41, no. 5, pp. 554-572, 2007.

[13] J. Li, B. Lin, Z. Sun, and X. Geng, "An estimation model of timevarying origin -destination flows in expressway corridors based on unscented Kalman filter," Science in China, Series E: Technological Sciences, vol. 52, no. 7, pp. 2069-2078, 2009.

[14] I. Okutani, "The kalman filtering approaches in some transportation and traffic problems," in Proceedings of the 10th International Symposium on Transportation and Traffic Theory, pp. 397-416, Elsevier Science, New York, NY, USA, 1987.

[15] K. Ashok and M. E. Ben-Akiva, "Alternative approaches for realtime estimation and prediction of time-dependent origindestination flows," Transportation Science, vol. 34, no. 1, pp. 2136, 2000.

[16] K. Ashok and M. E. Ben-Akiva, "Estimation and prediction of time-dependent origin-destination flows with a stochastic mapping to path flows and link flows," Transportation Science, vol. 36, no. 2, pp. 184-198, 2002.

[17] X. S. Zhou and H. S. Mahmassani, "A structural state space model for real-time traffic origin-destination demand estimation and prediction in a day-to-day learning framework," Transportation Research Part B, vol. 41, no. 8, pp. 823-840, 2007.

[18] H. D. Sherali and T. Park, "Estimation of dynamic origindestination trip tables for a general network," Transportation Research Part B: Methodological, vol. 35, no. 3, pp. 217-235, 2001.

[19] Y. Nie and H. M. Zhang, "A variational inequality formulation for inferring dynamic origin-destination travel demands," Transportation Research Part B: Methodological, vol. 42, no. 7-8, pp. 635-662, 2008.

[20] L. Cheng, S. Zhu, Z. Chu, and J. Cheng, "A Bayesian network model for origin-destination matrices estimation using prior and some observed link flows," Discrete Dynamics in Nature and Society, vol. 2014, Article ID 192470, 9 pages, 2014.

[21] S. Mikami and Y. Kakazu, "Genetic reinforcement learning for cooperative traffic signal control," in Proceedings of the 1st IEEE Conference on Evolutionary Computation, vol. 1, pp. 223-228, Orlando, Fla, USA, June 1994.

[22] G. Nakamiti and F. Gomide, "Fuzzy sets in distributed traffic control," in Proceedings of the 5th IEEE International Conference on Fuzzy Systems, pp. 1617-1623, New Orleans, La, USA, September 1996.

[23] E. Bingham, "Reinforcement learning in neurofuzzy traffic signal control," European Journal of Operational Research, vol. 131, no. 2, pp. 232-241, 2001.

[24] R.-M. Li and H.-P. Lu, "Traffic signal control multi-object optimization based on genetic algorithm," Journal of Chang'an University, vol. 29, no. 3, pp. 85-88, 2009.

[25] Z. Z. Yuan, Road Traffic Management and Control, China Communications Press, Beijing, China, 2007.

[26] J. Chang and D. Wu, "Multi-objective intersection signal control model," Journal of Dalian University of Technology, vol. 40, no. 6, pp. 653-656, 2000. 


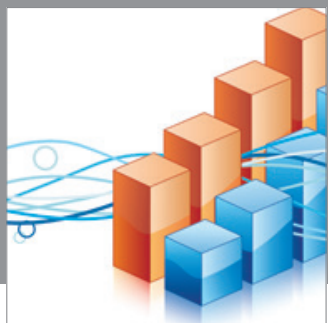

Advances in

Operations Research

mansans

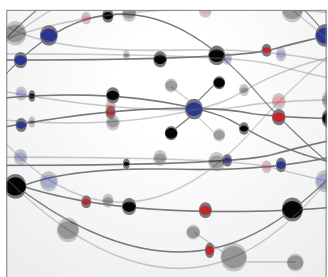

The Scientific World Journal
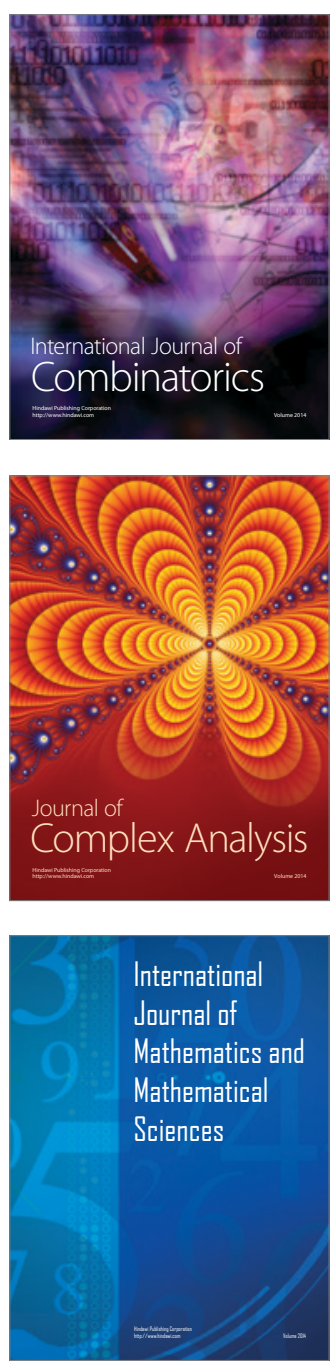
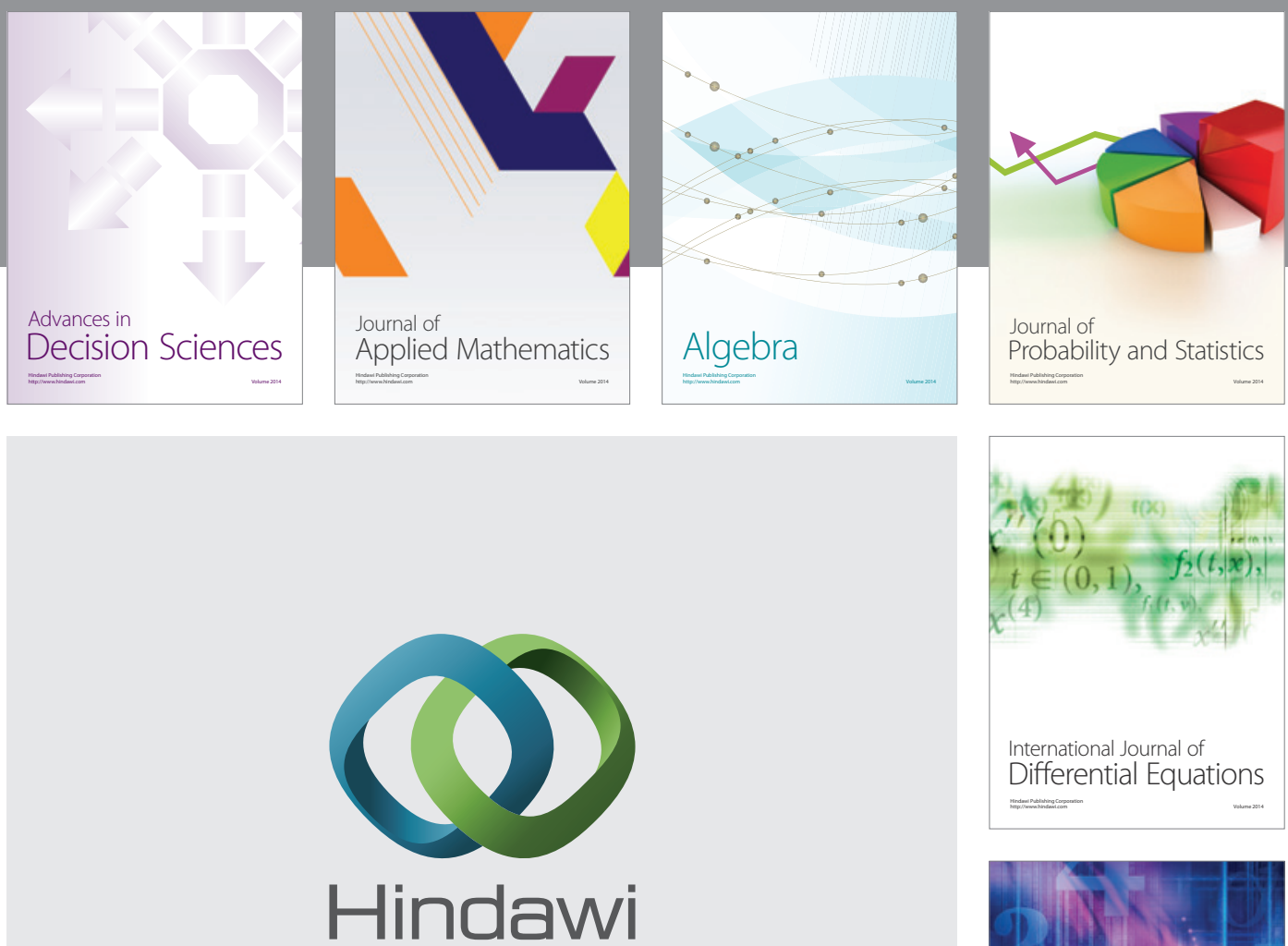

Submit your manuscripts at http://www.hindawi.com
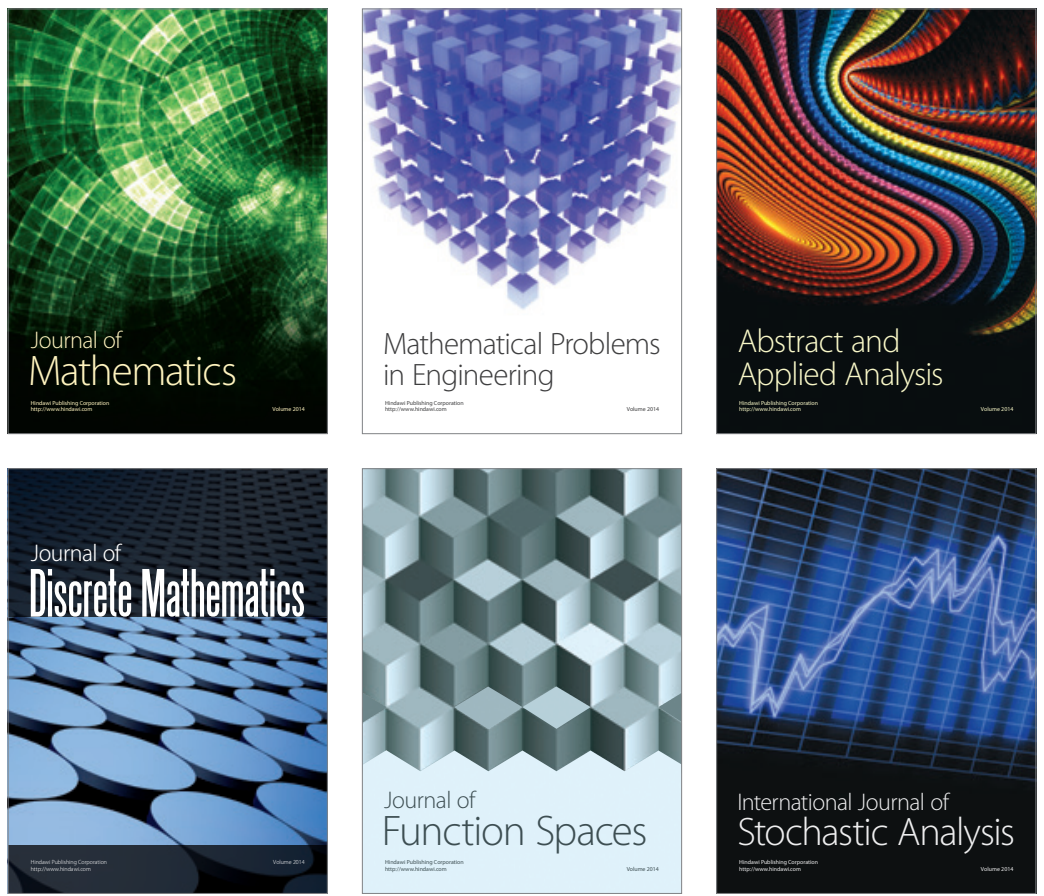

Journal of

Function Spaces

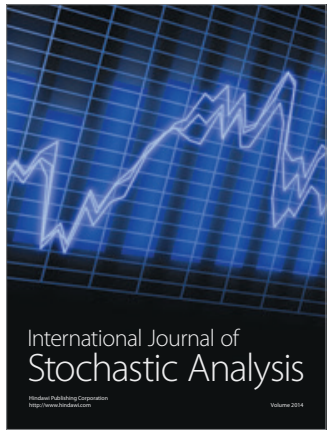

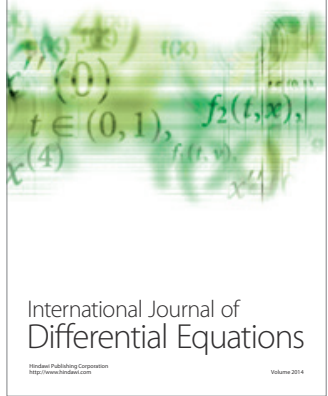
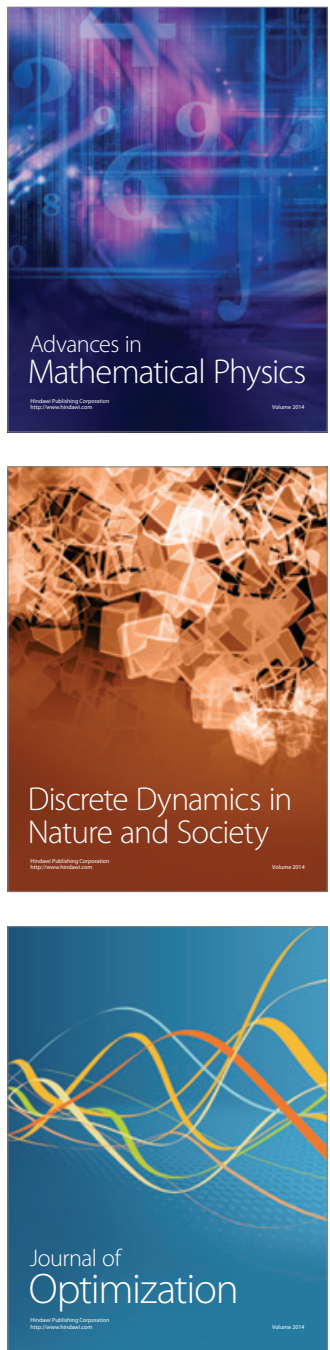